\title{
КУЛЬТУРНАЯ ВОСТРЕБОВАННОСТЬ СПОСОБНОСТЕЙ И ПСИХОМЕТРИЧЕСКИЕ СВОЙСТВА КОГНИТИВНЫХ ТЕСТОВ
}

\author{
Е.А. ВАЛУЕВА ${ }^{\mathrm{a}, \mathrm{b}}$, С.С. БЕЛОВА ${ }^{\mathrm{a}, \mathrm{b}}$, О.А. МОРОЗОВА ${ }^{\mathrm{a}}$ \\ ${ }^{a}$ Институт психологии РАН, 129366, Россия, Москва, ул. Ярославская, д. 13, стр. 1 \\ ${ }^{\circ}$ Московский государственный психолого-педагогический университет, 127051, Россия, Москва, \\ ул. Сретенка, д. 29
}

\section{Резюме}

В статье обсуждается проблема связи интеллекта с социокультурной средой и предлагается подход к этой проблеме в рамках структурно-динамической теории интеллекта. Центральный вопрос, вокруг которого вращается оппозищия биологизаторского и культурного подходов: каким образом происходит формирование интеллекта и какие факторы при этом являются главенствующими? Преимущество структурно-динамического подхода заключается в том, что он, принимая факты, на которых настаивают сторонники биологизаторского подхода, тем не менее переинтерпретирует их и показывает роль социальных и культурных факторов. Структурно-динамический подход использует понятия «когнитивный потенциал» и «культурная востребованность интеллектуальных функций». Когнитивный потенциал человека (отражающийся в генеральном факторе интеллекта - g) может быть направлен в различные сферы в зависимости от задач, выдвигаемых культурной и социальной средой. Когнитивный потенциал проявляется в большей степени в более востребованных средой способностях. В работе обсуждаются механизмы влияния среды на структуру интеллекта. Структура интеллекта характеризуется набором факторов, входящих в интеллект, связями между этими факторами и уровнем развития способностей, входяцих в различные факторы. Согласно предлагаемому подходу, социокультурная среда не влияет на набор факторов, входящих в структуру интеллекта, но изменяет связи факторов и показатели выборки по этим факторам. Приводятся эмпирические данные, подтверждающие эту точку зрения. Так, например, кросскультурные исследования демонстрируют инвариантность факторной структуры интеллекта в выборках из разных стран, но при этом низкую кросс-национальную валидность тестовых норм. Данные собственного исследования авторов на материале теста Векслера показывают, что ценность той или иной когнитивной способности в обществе связана с ее нагрузкой по генеральному фактору и показателями наследуемости.

Ключевые слова: интеллект, структура интеллекта, генетика, среда, культура.

Проблема интеллекта и культуры вызывает острые дискуссии как в плане фундаментальных научных позиций, так и в отношении практических следствий. Одна сторона этих дискуссий - биологизаторская -

Статья написана при поддержке совместного гранта РФФИ и Правительства Москвы, проект № 15-36-70009. 
рисует человеческий интеллект как определяемый преимущественно генетикой и интерпретирует данные о связи интеллекта с достижениями человека в современном обществе как указание на генетическую заданность этих достижений. Отсюда порой следуют призывы к проведению жесткой, лишенной гуманистической направленности социальной политики (Herrnstein, Murrey, 1994). Поскольку человеческие расы и нации имеют некоторые генетические особенности и эмпирические исследования показали различия в их когнитивных способностях, биологизаторская позиция в практическом и социальном планах нередко характеризуется оппонентами как расистская.

Противоположная точка зрения упор на культурной составляющей интеллекта и его средовой обусловленности. Сторонники этого подхода в своих исследованиях стараются показать, каким образом социальная среда формирует интеллект, в том числе - компенсируя генетические различия. Такая позиция гораздо привлекательнее в социальном плане. Она делает акцент на ответственности человека и общества и препятствует стигматизации неудачников.

Однако как бы ни были социально и практически привлекательны те или иные научные позиции, центральным критерием остается их соответствие истине. Здесь же картина продолжает оставаться крайне запутанной, чему способствует и происходящее порой смешение собственно научных аргументов с ценностными. Культурный подход пока добился весьма скромных успе- хов в эмпирическом обосновании своих концепций. Ряд данных высокие показатели наследуемости интеллекта, полученные в близнецовых исследованиях, «коррелирующие вектора» наследуемости и межрасовых различий и т.д. - легко включаются в биологизаторские схемы, но плохо поддаются объяснению в рамках культурного подхода.

Ключевой методологический момент в этом споре заключается в том, что его разрешение может достигаться не на уровне фактов, а лишь через модели, которые, как известно после К. Поппера, фактами не доказываются, а лишь фальсифицируются. Возьмем, например, данные близнецовых исследований, которые дают оценку наследуемости интеллекта в 50-80\% при весьма скромном эффекте общей среды. Эти результаты хорошо обоснованы и надежно получены. На поверхности, т.е. при использовании простейших объяснительных схем, они кажутся подтверждением биологизаторского подхода. Однако при углублении теоретического взгляда оказывается, что это отнюдь не так.

В самом деле, данные близнецового исследования зависят от генотипического разнообразия изучаемой популяции. Если изменить это разнообразие, результаты получатся иными. Например, если работать с популяцией клонов, т.е. ликвидировать генетическую вариативность, то генетика не будет оказывать никакого влияния на дисперсию поведения индивидов в выборке. Генетика поведения констатирует в этом случае, что коэффициент наследуемости равен нулю. Но, конечно же, при этом не перестанут действовать механизмы экспрессии генов, синте- 
за белков, приводящие к формированию клеток с определенными свойствами, а значит, и поведенческих особенностей.

Если же представить, что могущественный экспериментатор создал для индивидов, входящих в некую выборку, строго одинаковые условия ${ }^{1}$, то влияние среды исчезнет и поведенческая генетика примет наследуемость за 100\%. Однако для индивида не изменится ничего - его процессы будут функционировать точно так же, как они функционировали бы, если бы у других индивидов условия были другими. Таким образом, одни и те же молекулярно-генетические процессы на уровне индивида при различном составе выборки и средовых условиях приведут к различным (а иногда и диаметрально противоположным) оценкам на уровне генетики поведения.

Сказанное означает, что эмпирически полученные цифры наследуемости интеллекта, будучи абсолютно правильными и надежными, оказываются в то же время «лукавыми», поскольку их смысл оказывается зависимым от глубины интерпретации, т.е. от теоретической проработанности объяснительных моделей.

Отсюда становится очевидным центральное значение развития объяснительных моделей, которые могли бы, хорошо соответствуя фактам, в то же время придать им смысл, в частности, социальный и практический.

В этой статье будет предложен подход к проблеме, получивший название структурно-динамического (Ушаков, 2011). Преимущество этого подхода заключается в том, что он, принимая фактологию, на которой настаивают сторонники биологизаторского подхода, тем не менее переинтерпретирует ее и направляет в русло, где становится отчетливо видна роль социальных и культурных факторов.

\section{Структура интеллекта и ее детерминанты}

Фактически центральный вопрос, вокруг которого врашается оппозиция биологизаторского и культурного подходов, состоит в том, каким образом происходит формирование интеллекта и какие факторы при этом являются главенствующими. Для того чтобы рассмотреть этот вопрос со всей серьезностью, бесполезно анализировать интеллект как нечто единое, а нужно обратиться к его структуре.

Согласно современным представлениям, интеллект, или совокупность когнитивных способностей, имеет многоуровневую иерархиче-

\footnotetext{
${ }^{1}$ Конечно, практически осуцествление такого әксперимента невозможно не только на человеке, но и на животных. Строго одинаковые условия в указанном выше смысле должны исключить и генно-средовое взаимодействие, т.е. не должны допускать изменения среды под воздействием индивидуальности субъекта. Если индивид может влиять на среду, то его индивидуальные особенности так изменяют среду, что она в свою очередь создает для индивида условия, отличаюшиеся от условий для других индивидов. В этом смысле даже не всякая виртуальная компьютерная среда создает строго одинаковые условия, поскольку во многих случаях изменяется от действий субъекта.
} 
скую структуру, увенчанную генеральным фактором, или фактором g. На рисунке 1 представлена наиболее влиятельная и объемлющая на сегодня модель, предложенная Дж. Кэрроллом (Carroll, 1993).

Изображенная на рисунке 1 модель представляет интеллект как трехуровневое образование. На верхнем уровне находится генеральный фактор, на промежуточном - 8 широких факторов, а на нижнем - 69 узких факторов. Понятие фактора означает, что входящие в него когнитивные способности коррелируют между собой. Самые высокие корреляции у тех способностей, которые входят в один и тот же узкий фактор. Несколько слабее коррелируют способности, входящие в один и тот же широкий фактор. Наличие генерального фактора знаменует тот факт, что все когнитивные способности в конечном счете положительно коррелируют между собой, т.е. то, что Ч.
Спирмен обозначил как «положительную множественность» (positive manifold).

Модель на рисунке 1 не является единственно возможной на сегодня, предлагаются и альтернативные интерпретации. Однако все они фактически сходятся в признании иерархической многоуровневости и генерального фактора, хотя различаются во взглядах на число уровней, конкретный перечень факторов и некоторые другие детали.

Понимание интеллекта как сложной структуры уже значительно продвигает в глубину дебаты о характере обусловленности интеллекта, однако чисто описательная характеристика, предоставляемая структурными моделями, по-прежнему оказывается недостаточной. Чтобы двигаться дальше, необходимо понимать, какие процессы, или механизмы, стоят за различными факторами интеллекта, что можно осуществить на основе

Модель структуры интеллекта Дж. Кәрролла

Puсунок 1

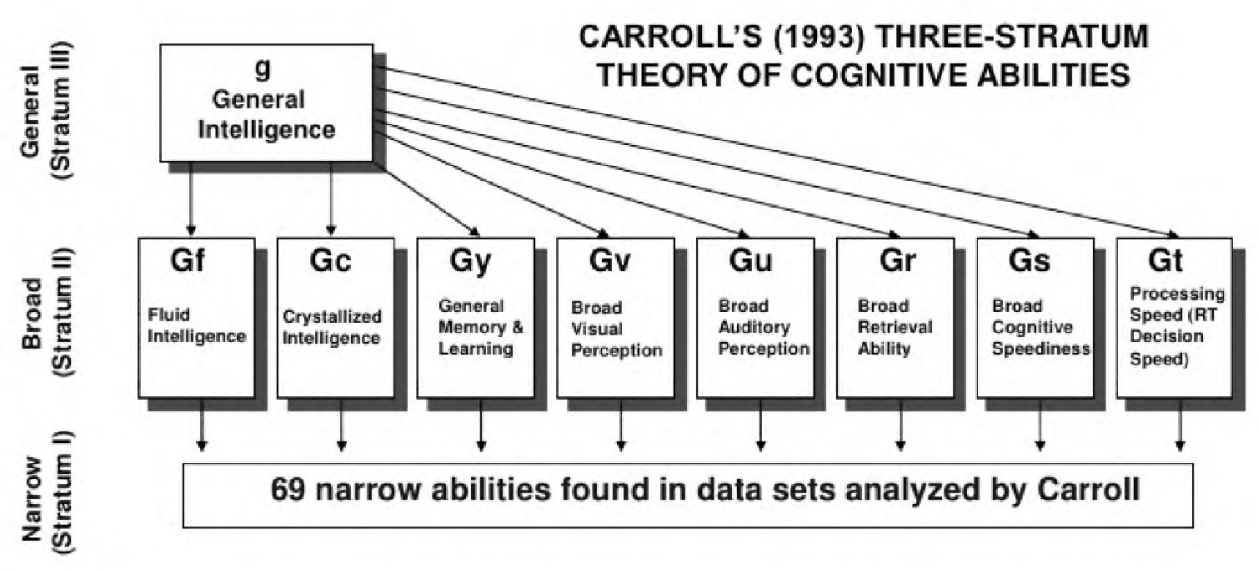

Примечание. Изображение взято с сайта https://sapa-project.org/blogs/CHCmodel.html 
структурно-динамической теории интеллекта.

Согласно структурно-динамической теории, генеральный фактор, в отличие от всех прочих, имеет иную природу. За узкими и широкими факторами интеллекта стоят отдельные процессы переработки информации или набор таких процессов, применяемых для решения сходных задач. Например, в одном влиятельном исследовании аргументируется, что флюидный интеллект (на примере матриц Равена) связан с «общей для всех испытуемых способностью раскладывать проблему на доступные для работы (manageable) части и поочередно заниматься ими, а также индивидуально выраженной способностью управляться с иерархией целей и подцелей, образованной разложением проблемы, и формировать абстракции высокого уровня» (Carpenter et al., 1990, p. 429). Аналогично можно выявить различные когнитивные компоненты, стоящие за вербальным интеллектом: извлечение информации из долговременной памяти, преобразование пропозициональной репрезентации в аналоговую и т.д.

Другое дело - генеральный фактор. Он стоит за корреляциями, которые связывают между собой решение задач, использующих совершенно различные наборы когнитивных процессов. Поэтому его трудно отождествить как с каким-либо одним когнитивным процессом, так и даже с набором таких процессов. Согласно структурно-динамической теории, генеральный фактор отражает когнитивный потенциал человека, который может быть направлен в различные сферы в зависимости от задач, выдвигаемых культурной и социальной средой.

\section{Культурные влияния на структуру интеллекта}

Из изложенных взглядов структурно-динамической теории вытекает предположение о том, каким образом социокультурное влияние может отражаться на структуре интеллекта. Структура интеллекта характеризуется несколькими чертами. Во-первых, это набор факторов. Например, набор факторов структуры интеллекта, изображенной на рисунке 1 , составляют генеральный фактор, восемь широких факторов и шестьдесят девять узких. Во-вторых, связи между факторами. На рисунке 1 обозначены только сами эти связи, без спецификации их силы. Так, генеральный фактор влияет на широкие факторы, а каждый из последних - на целую серию связанных узких факторов. Между тем сила связей может быть различной. Такой статистический метод, как конфирматорный факторный анализ, позволяет специфицировать силу связей. Структуры, состоящие из одного и того же набора факторов, имеющих связи разного вида или одного вида, но разной силы, не являются идентичными.

Наконец, даже при одинаковых структурах выборки могут различаться по уровню развития способностей, входящих в различные факторы. При этом одна выборка может уступать другой по уровню развития одних способностей, но превосходить по развитию других.

С этих позиций следует взглянуть на предсказания структурно-дина- 
мической теории интеллекта в отношении культурных влияний на структуру интеллекта и сопоставить эти предсказания с эмпирическими данными. Набор факторов, входящих в структуру интеллекта, определяется совокупностью когнитивных процессов, которые разворачиваются при решении различных задач. Следовательно, можно ожидать, что набор факторов остается инвариантом при рассмотрении выборок, рекрутированных в различных культурных средах.

Действительно, эмпирические исследования подтверждают такое предположение.

В исследовании Дж. Лиу и Р. Линна (Liu, Lynn, 2011) на выборках 5-6-летних детей изучались факторная структура детского варианта теста Векслера (the Wechsler Preschool and Primary Scale of Intelligence, WPPSI), а также половые и кросс-культурные различия. В трех культурах выявлена инвариантность факторной структуры с двумя факторами - вербальным и невербальным. Данные о половых различиях были в целом консистентны и заключались в преимуществе мальчиков по выполнению пространственного субтеста (субтест лабиринты). Мальчики китайской выборки имели статистически значимый более высокий балл, чем девочки, и меньшую вариативность тестовых баллов. Такие результаты не были отмечены в японской и американской выборках и нашли у авторов культурологическое объяснение: в Китае сохраняется больший пиетет по отношению к мужчинам, что проявляется в особенностях раннего образования, предоставляющего мальчиками обогащенную среду, лучшие ресурсы и т.д. Меньшая вариативность в баллах мальчиков также рассматривается авторами как культурно-специфическое явление, не характерное для наблюдаемых на Западе данных, где картина является обратной (для мальчиков характерная большая вариативность). Среднее значение общего балла по тесту составляло в Китае 104.1, что соответствовало росту в 4 балла с 1998 по 2004 г.

Если в отношении набора факторов структурно-динамическая теория предсказывает независимость результатов от культурной среды исследуемой выборки, то в отношении силы связи факторов предсказание иное. А именно повышение ценности какой-либо интеллектуальной функции в обществе и следующее за ним увеличение тренировки членов общества в соответствуюшей сфере должно приводить к увеличению нагрузки генерального фактора на эту функцию.

Эти предсказания получили подтверждение в исследовании авторов, выполненном на материале теста Векслера. Было показано, что субшкалы этого теста, тестирующие более ценные в обществе функции, оказываются более высоко нагруженными генеральным фактором и демонстрируют более высокие индексы наследуемости (Валуева, Ушаков, 2013).

В плане дальнейшей проверки этого предсказания интерес представляет сопоставление факторных структур, полученных по результатам исследования в различных культурах и в различные временны́е промежутки.

Наконец, структурно-динамическая теория признает, что культура 
может оказывать стимулирующие влияние на отдельные когнитивные процессы и, следовательно, повышать результаты по отдельным факторам, входящим в структуру интеллекта. Существующие на сегодняшний день эмпирические данные согласуются с такой трактовкой. Так, при сравнении американских и европейских норм по субтестам Векслера WAIS III выявляется, что при переводе европейских сырых данных в оценки интеллекта с использованием американских норм средние значения индекса перцептивной организации (POI) оказываются значительно выше средних значений индекса скорости переработки (PSI). Соотношение POI/PSI составляет примерно 5-10 баллов для выборок стандартизации Германии $(\mathrm{n}=1897)$, Испании (n = 1369), Франции $(\mathrm{n}=104)$, Британии $(\mathrm{n}=370)$, Финляндии ( $\mathrm{n}=446)$ (Roivainen, 2010). Среднее значение индекса POI выше во всех группах европейской выборки. Сделано заключение: маловероятно, что эти эффекты связаны с действием одного фактора (фактор общего интеллекта). Выдвигается гипотеза, что в основе различий между американским и европейским профилем способностей лежат культурные факторы, различия в образовательных системах и установки по отношению к тестированию. Согласно результатам исследования, кросс-национальная валидность национальных тестовых норм невелика даже при контроле эффектов возраста, пола и образования испытуемых.

Культурно-обусловленные установки по отношению к тестированию - одно из возможных объясне- ний кросс-атлантических различий в отношении POI/PSI. Было высказано предположение, что быстрое исполнение является важной культурной ценностью в США, но менее ценным в других культурах, что ставит под угрозу кросс-национальную валидность когнитивных и нейробиологических измерений (Rosselli, Ardila, 2003; Leon-Carrion, 1989; Boivin et al., 1995; Agranovich, Puente, 2007). Такие данные сушествуют в отношении детей Испании, России, Заира. Результаты сравнения выборок из стран Европы и США подводят даже к определению в качестве «коррелята» ценности высокой скорости то, что популярные в США виды спорта связаны с быстротой реагирования и нападением, в то время как европейская приверженность «скучному» футболу, когда полуторачасовой матч заканчивается со счетом 1:0, трактуется как принципиально иная установка по отношению к временни́м ресурсам (Roivainen, 2010). Фастфуд, работа в течение короткого времени, короткие отпуска - также примеры культурно-специфичной американской скорости.

\section{Выводы}

Приведенные данные показывают, что модель на основе структурно-динамической теории способна предложить достаточно дифференцированные предсказания, которые неплохо сходятся с эмпирическими данными. В то же время структурнодинамическая модель открывает место для культурно-исторического понимания интеллекта и позволяет избежать прямолинейности, свой- 
ственной биологизаторскому подходу. Такие показатели, как коэффициент наследуемости или нагрузка по генеральному фактору, приобретают другое звучание: они начинают пониматься не в качестве вечного и неизменного соотношения, а как специфические показатели, характеризующие функционирование когнитивной системы человека в исторически особенной социокультурной среде.

\section{Литература}

Валуева, Е. А., Ушаков, Д. В. (2013). Культурная релевантность и свойства тестов интеллекта: проверка предсказаний структурно-динамической теории. Психолоzия. Журнал Высией иколы экономики, 10(3), 29-40.

Ушаков, Д. В. (2011). Психология интеллекта и одаренности. М.: Изд-во «Институт психологии РАН».

Ссылки на зарубежные источники см. в разделе References после англоязыиного блока.
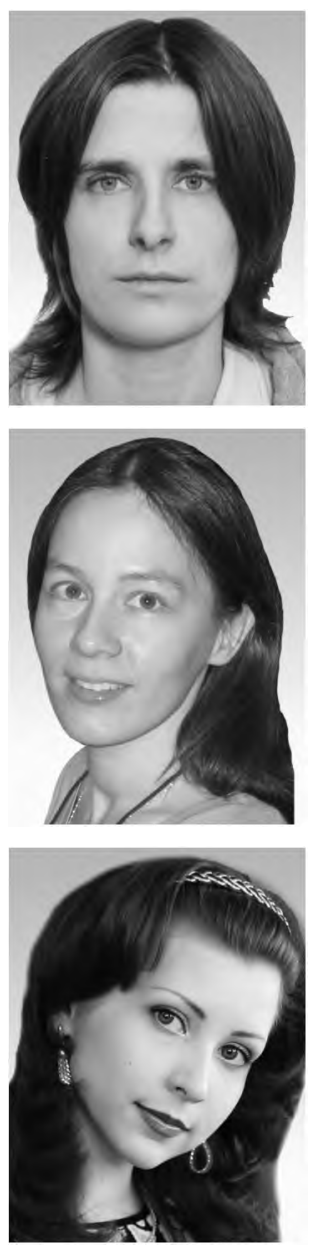

Валуева Екатерина Александровна - научный сотрудник, ФГБУН Институт психологии РАН; ведущий научный сотрудник, ГБОУ ВПО Московский государственый психолого-педагогический университет, кандидат психологических наук.

Сфера научных интересов: интеллект, креативность, когнитивные механизмы инкубации.

Контакты: ekval@list.ru

Белова Софья Сергеевна - научный сотрудник, ФГБУН Институт психологии РАН; ведущий научный сотрудник, ГБОУ ВПО Московский государственый психолого-педагогический университет, кандидат психологических наук.

Сфера научных интересов: интеллект, креативность, имплицитное научение, вербальные способности, социальное познание.

Контакты: sbelova@gmail.com

Морозова Ольга Андреевна - аспирант, ФГБУН Институт психологии РАН.

Сфера научных интересов: математическое моделирование, наука о сложных сетях, ментальный лексикон, психология интеллекта, психология видеоигр.

Контакты: helgamoro@gmail.com 


\title{
Cultural Relevance of Abilities and Psychometric Properties of Cognitive Tests
}

\author{
E.A. Valueva ${ }^{a, b}$, S.S. Belova ${ }^{a, b}$, O.A. Morozova ${ }^{a}$ \\ ${ }^{a}$ Institute of Psychology, Russian Academy of Sciences, 13 build. 1, Yaroslarskaya str., Moscow, 129366, \\ Russian Federation \\ ${ }^{b}$ Moscow State University of Psychology and Educaion, 29 Sretenka Str., Moscoze, 127051, Russian \\ Federation
}

\begin{abstract}
The article discuss the issue of relations between intelligence and socio-cultural environment. The subject is approached in the framework of structural dynamic theory of intelligence. The central question of biological and cultural approaches is "How is the intelligence formed and which factors are the pivotal?" The advantage of the structural dynamic approach is that while it accepts the facts of the biological approach, it reinterprets them and shows the role of social and cultural factors. The structural dynamic approach uses concepts "cognitive potential" and "cultural relevance of intellectual functions". The cognitive potential of an individual (as reflected in the general factor of intelligence $-\mathrm{g}$ ) may be allocated to various spheres, depending on tasks that are imposed by cultural and social environment. The cognitive potential is manifested to a greater degree in abilities, which are more demanded by the environment. The article discusses the mechanisms of influence of the environment on the structure of intelligence. The structure of intelligence in characterized by the set of factors, included into g, by the relations between those factors, and by the level of development of abilities, which are constitute various factors. According to the proposed approach, the socio-cultural environment does not influence the set of factors, included in the structure of intelligence, but it changes the relations between the factors, and the scores of these factors in the sample. The empirical data is provided that proves this perspective. Thus, for example, cross-cultural studies show invariance of the factor structure in the samples from different countries, but low cross-national validity of the test norms. The data of the authors' research with Wechsler test shows that the value in the society of any given cognitive ability is linked with its loading on the general factor and the indices of heritability.
\end{abstract}

Keywords: intelligence, structure of intelligence, genetics, environment, culture.

\section{References}

Agranovich, A. V., \& Puente, A. E. (2007). Do Russian and American normal adults perform similarly on neuropsychological tests? Preliminary findings on the relationship between culture and test performance. Archives of Clinical Neuropsychology, 22, 273-282.

Boivin, M. J., Giordani, B., \& Bornefeld, B. (1995). Use of the tactual performance test for cognitive ability testing with African children. Neuropsychology, 9, 409-417. 
Carpenter, P. A., Just, M., \& Shell, P. (1990). What one intelligence test measures: A theoretical account of processing in the Raven's progressive matrices test. Psychological Review, 97, 404-431.

Carroll, J. B. (1993). Human cognitive abilities: A survey of factor-analytic studies. Cambridge, UK: Cambridge University Press.

Herrnstein, R. J., \& Murray, C. (1994). Bell curve: Intelligence and class structure in American life. New York: Free Press.

Leon-Carrion, J. (1989). Trail making test scores for normal children: Normative data from Spain. Perceptual and Motor Skills, 68, 627-630.

Liu, J., \& Lynn, R. (2011). Factor structure and sex differences on the Wechsler Preschool and Primary Scale of Intelligence in China, Japan and United States. Personality and Individual Differences, 50(8), 1222-1226. doi:10.1016/j.paid.2011.02.013

Roivainen, E. (2010). European and American WAIS III norms: Cross-national differences in performance subtest scores. Intelligence, 38(1), 187-192. doi:10.1016/j.intell.2009.10.001

Rosselli, M., \& Ardila, A. (2003). The impact of culture and education on non-verbal neuropsychological measurements: A critical review. Brain and Cognition, 52(3), 326-333. doi:10.1016/S02782626(03)00170-2

Ushakov, D. V. (2011). Psikhologiya intellekta i odarennosti [The psychology of intelligence and giftedness]. Moscow: Institute of Psychology of Russian Academy of Sciences.

Valueva, E. A., \& Ushakov, D. V. (2013). Cultural relevance and properties of intelligence measures: Testing the structural-dynamic theory. Psychology. Joumal of Higher School of Economics, 10(3), 29-40. (in Russian)

Ekaterina A. Valueva - research fellow, Institute of Psychology, Russian Academy of Sciences; senior research fellow, Moscow State University of Psychology \& Education, Ph.D.

Research area: intellect, creativity, cognitive mechanisms of incubation.

Email: ekval@list.ru

Sofya S. Belova - research fellow, Institute of Psychology, Russian Academy of Sciences; senior research fellow, Moscow State University of Psychology \& Education, Ph.D.

Research area: intellect, creativity, implicit learning, verbal abilities, social cognition.

Email: sbelova@gmail.com

Olga A. Morozova - PhD student, Institute of Psychology, Russian Academy of Sciences.

Research area: mathematical models, complex network science, mental lexicon, psychology of intellect, psychology of videogames.

E-mail: helgamoro@gmail.com 